\title{
The Positivstellensatz and Nonexistence of Common Quadratic Lyapunov Functions
}

\author{
John M. Davis and Geoffrey Eisenbarth \\ Department of Mathematics \\ Baylor University \\ Waco, TX 76798 \\ Email: John_M_Davis@baylor.edu, \\ Geoffrey_Eisenbarth@baylor.edu
}

\begin{abstract}
We provide an algorithm for establishing the nonexistence of a common quadratic Lyapunov function for switched LTI systems under arbitrary switching. We show that this nonexistence question is equivalent to the emptiness of an associated semi-algebraic set. The celebrated Positivstellensatz from real algebraic geometry provides a complete characterization of when this set is empty. Finally, we obtain the desired certificates of set emptiness using sum of squares programming.
\end{abstract}

Index Terms-Positivstellensatz, common Lyapunov functions, sum of squares, switched systems, real algebraic geometry

\section{INTRODUCTION}

Stability of switched systems can be determined by the identification of a single quadratic Lyapunov function applicable to all component systems. These common quadratic Lyapunov functions (CQLF) and their nonexistence has been discussed in several papers [3], [4], [5], [7]. In this paper, we outline an algorithm for determining nonexistence of a CQLF based on methods from real algebraic geometry.

We begin by recalling definitions from linear time invariant (LTI) switched system theory and the motivation behind finding a CQLF for the system. Next, we define the concepts needed from real algebraic geometry, namely Stengle's Positivstellensatz, which is an analog of Hilbert's classical Nullstellensatz. We then convert the CQLF existence problem to system of simultaneous polynomial inequalities and use techniques from real algebraic geometry to determine whether the polynomial system has a solution. We accomplish this by relating the Positivstellensatz to a sum of squares (SoS) program along the lines of Parrilo's work [8], [9]. When successful, the SoS program shows that there does not exist a simultaneous solution to the polynomial system which in turn implies the nonexistence of a CQLF. Finally, we outline this entire process for a particular switched system and illustrate the usefulness of certain MATLAB toolboxes for performing the SoS programming.

\section{Stability of Switched Systems}

A linear $N$-switched system is comprised of a switching signal $s(t): \mathbb{R} \rightarrow\{1, \ldots, N\}$ and a set of LTI systems $\dot{x}(t)=A_{i} x(t)$, where $1 \leq i \leq N$; which we write as $\dot{x}=A_{s(t)} x$. Stability for switched systems under arbitrary switching requires stronger conditions than just the component systems being stable; it has been noted [5] that even if all subsystems are stable it is possible to switch between the systems in a manner that would produce unstable solutions.

One method of proving the stability of switched systems is by finding a CQLF for the system, which has been studied extensively. We say $V: \mathbb{R}^{n} \rightarrow \mathbb{R}$ is a common quadratic Lyapunov function if $V(x)=x^{T} P x$ is positive definite and $\dot{V}(x)=A_{i}^{T} P+P A_{i} \leq 0$ for all $1 \leq i \leq N$. In particular, Narendra and Balakrishnan [6] showed that a sufficient condition on the matrices $A_{i}$ to guarantee the existence of a CQLF under arbitrary switching is that the $A_{i}$ are stable (eigenvalues in the strict left half complex plane) and pairwise commutative. Relaxing these types of commutativity assumptions in terms of the Lie algebra generated by the $A_{i}$ was dealt with extensively in [1].

Although the Narendra-Balakrishnan result requires pairwise commuting $A_{i}$, we investigate the existence of a CQLF without the pairwise commuting stipulation. In particular, we provide a means to generate a proof of the nonexistence of a CQLF for a given switched system.

Throughout this paper, we appeal to Sylvester's Criterion [2, Theorem 7.5.2] of positive definiteness: a real symmetric matrix is positive definite if and only if all of its leading principal minors have positive determinant. For an $n \times n$ real symmetric matrix, this results in $n$ polynomial inequalities as shown in the example.

Example II.1. Consider the LTI system $\dot{x}=A x$ where

$$
A=\left[\begin{array}{ll}
a_{1} & a_{2} \\
a_{3} & a_{4}
\end{array}\right]
$$

From standard Lyapunov theory, $V=x^{T} P x$ is a Lyapunov function for this system provided $P=P^{T}>0$ and $A^{T} P+$ $P A \leq 0$. Letting

$$
P=\left[\begin{array}{ll}
x & y \\
y & z
\end{array}\right]
$$

and employing Sylvester's Criterion to the symmetric matrix $-\left(A^{T} P+P A\right)$, these two conditions are equivalent to the 
following system of polynomial inequalities:

$$
\begin{aligned}
x & >0, \\
x z-y^{2} & >0, \\
-2\left(a_{1} x+a_{3} y\right) & \geq 0, \\
-4\left(a_{1} x+a_{3} y\right)\left(a_{2} y+a_{4} z\right) & \\
+\left[\left(a_{1}+a_{4}\right) y+a_{2} x+a_{3} z\right]^{2} & \geq 0 .
\end{aligned}
$$

To extend this example to a switched system involving $A_{i}, i=$ $1, \ldots, N$, note that the corresponding system of polynomial inequalities will have the same basic structure as (II.1). When $A_{i} \in \mathbb{R}^{2 \times 2}$, this introduces $2 N$ new inequalites each of the form of the last two above. In general, when $P$ is symmetric and $A_{i} \in \mathbb{R}^{n \times n}$, the polynomial system will consist of $1+$ $2+\cdots+n=\frac{1}{2} n(n+1)$ unknowns.

\section{Real Algebraic Geometry}

Real algebraic geometry is concerned with the interplay of sets defined by polynomials with real-valued coefficients and the underlying geometry of said sets; oftentimes algebraic problems can be easily solved when cast in a geometric light and vice versa. In particular, we focus on the aspects of real algebraic geometry which relate the solvability of a simultaneous system of polynomial inequalities with the emptiness of the associated semi-algebraic set (defined below).

We will denote by $\mathbb{R}\left[x_{1}, \ldots, x_{n}\right]=\mathbb{R}[X]$ the ring of polynomials in $n$ unknowns with real valued coefficients.

Definition III.1. Given $\left\{f_{i}\right\}_{i \in I} \subset \mathbb{R}[X]$, a (real) semialgebraic set is a set of the form

$$
\left\{x \in \mathbb{R}^{n}: f_{i}(x) * 0 \forall i \in I\right\},
$$

where $*$ represents any of the following: $\geq, \neq$, or $=$.

Example III.1. Let $\left\{f_{1}(x, y):=x^{2}, f_{2}(x, y):=x y^{2}\right\} \subset$ $\mathbb{R}[x, y]$. Then

$$
\left\{(x, y) \in \mathbb{R}^{2}: f_{1}(x, y) \geq 0, f_{2}(x, y) \neq 0\right\}
$$

is a semi-algebraic set, whereas

$$
\left\{(x, y) \in \mathbb{R}^{2}: f_{1}(x, y)=e^{-x} \sin y \geq 0\right\}
$$

is not a semi-algebraic set.

Many types of problems can be formulated through semialgebraic sets. Primarily in this paper, we will be concerned with when a given semi-algebraic set is empty, and the Positivstellensatz is a tool that can assist us in this goal. Before we state the theorem in our formulation, we must define three more concepts.

Definition III.2. Given a set $G \subset \mathbb{R}[X]$ of polynomials, we define the monoid generated by $G$ to be the set

$$
\operatorname{monoid}\{G\}:=\left\{g_{1}^{m_{1}} \ldots g_{n}^{m_{n}}: g_{i} \in G, m_{i} \in \mathbb{N}_{0}\right\} .
$$

with the usual commutative multiplication on $\mathbb{R}[X]$.
Example III.2. Let $G=\left\{g_{1}(x, y):=x+y, g_{2}(x, y):=\right.$ $y\} \subset \mathbb{R}[x, y]$. Then

$$
\operatorname{monoid}\{G\}=\left\{(x+y)^{n} y^{m}: n, m \in \mathbb{N}_{0}\right\} .
$$

Notice that this contains $f(x, y)=(x+y)^{n}$ and $h(x, y)=y^{m}$ for all natural numbers $n$ and $m$.

Definition III.3. The cone of sum of squares polynomials, denoted $\Sigma^{2}$, is given by

$$
\Sigma^{2}:=\left\{f \in \mathbb{R}[X]: f(X)=\sum g_{i}^{2}(X), g_{i}(X) \in \mathbb{R}[X]\right\} .
$$

Given $F \subset \mathbb{R}[X]$, we define the cone generated by $F$ as

$$
\begin{aligned}
\operatorname{cone}\{F\}:=\left\{s_{0}+s_{1} f_{1}+\cdots+s_{n} f_{n}:\right. \\
\left.f_{i} \in \operatorname{monoid}\{F\}, s_{i} \in \Sigma^{2}\right\} .
\end{aligned}
$$

It is important to note that a general member of the set cone $\{F\}$ can be considered as a sum of square polynomial $s_{0}$ plus all possible multiples (without repetition) of elements in $F$ times $\mathrm{SoS}$ polynomials. While the definition allows for terms such as $s_{1} f_{1}^{2} f_{2}^{3} f_{4} f_{8}$ to be in cone $\{F\}$ (where $f_{1}, f_{2}, f_{4}, f_{8} \in F$, and the superscript refers to exponentiation), notice that $s_{1} f_{1}^{2} f_{2}^{3} f_{4} f_{8}=s_{1} f_{1}^{2} f_{2}^{2} f_{2} f_{4} f_{8}$, and $s_{1} f_{1}^{2} f_{2}^{2} \in$ $\Sigma^{2}$. Therefore we could represent this term by using the sum of square $s_{2}=s_{1} f_{1}^{2} f_{2}^{2}$, as in $s_{2} f_{2} f_{4} f_{8}$.

Example III.3. Let $F=\left\{f_{1}, f_{2}\right\} \subset \mathbb{R}[X]$. Then any element in cone $\{F\}$ can be represented in the form

$$
s_{0}+s_{1} f_{1}+s_{2} f_{2}+s_{3} f_{1} f_{2},
$$

where $s_{i} \in \Sigma^{2}$.

We can now state a formulation of the Positivstellensatz ${ }^{1}$ suitable for our purposes.

Theorem III.1 (Positivstellensatz, [10]). Let $F, G \subset \mathbb{R}[X]$. Then the semi-algebraic set

$$
\left\{x \in \mathbb{R}^{n}: f(x) \geq 0 \forall f \in F, g(x) \neq 0 \forall g \in G\right\}
$$

is empty iff there exists $f \in \operatorname{cone}\{F\}$ and $g \in \operatorname{monoid}\{G\}$ such that $f+g^{2}=0$.

The goal now is to use the Positivstellensatz to determine whether the semi-algebraic set generated by the polynomial inequalities resulting from the conditions on the CQLF is empty. A key component of the argument is that the search for $f \in \operatorname{cone}\{F\}$ and $g \in \operatorname{monoid}\{G\}$ such that $f+g^{2}=0$ is equivalent to searching for certain types of SoS polynomials.

To illustrate this, suppose we want to determine if the set

$$
\left\{X \in \mathbb{R}^{n}: f_{1}(X) \geq 0, f_{2}(X) \geq 0, g_{1}(X) \neq 0\right\}
$$

is empty or not. By Example III.3, a general element in cone $\left\{f_{1}, f_{2}\right\}$ is of the form $s_{0}+s_{1} f_{1}+s_{2} f_{2}+s_{3} f_{1} f_{2}$, so we need to determine if there exist SoS polynomials $s_{i}$ such that

$$
s_{0}+s_{1} f_{1}+s_{2} f_{2}+s_{3} f_{1} f_{2}+g_{1}^{2 m}=0,
$$

\footnotetext{
${ }^{1}$ The name of this theorem comes from the German for "positive places theorem" since it determines the subset of $\mathbb{R}^{n}$ on which a system of polynomials is positive.
} 
for some $m \in \mathbb{N}$.

To recapitulate, we took the problem of determining the existence of a CQLF and translated it to a problem involving the simultaneous solution to a system of polynomial inequalities. We then recast that as a problem of determining whether the associated semi-algebraic set was empty. We will resolve this "set emptiness question" via the Positivstellensatz and do so by looking for $f$ and $g$ with the SoS representations described above.

\section{SOS PROGRAMMING AND SOSTOOLS}

An SoS program is a special case of a semi-definite program (SDP), which is a generalization of linear programming. In an SDP, one searches over the cone of positive semi-definite matrices as opposed to the set of coordinate-wise non-negative numbers. We only offer a brief overview of the relationship between real algebraic geometry and SDP here, but the interested reader should consult [8].

An SoS program has the following form:

$$
\begin{gathered}
\text { Given } a_{i, j} \in \mathbb{R}[X] \text {, find SoS polynomials } s_{i}(X) \text { such that } \\
\qquad a_{0, j}+\sum_{i=1}^{n} a_{0, i} s_{i}=0, j=1, \ldots, m .
\end{gathered}
$$

This is useful since in order to obtain an answer from the Positivstellensatz, we must find SoS polynomials such that $f+$ $g^{2}=0$. If the constraint equality in the SoS program is given by $f+g^{2}=0$, then solving the SoS program is equivalent to proving that no CQLF exists for the switched system. Since the Positivstellensatz is both necessary and sufficient, if the semi-algebraic set is indeed empty, then the SoS program will eventually find certificates of emptiness (the certificates being the $\left.s_{i}(X) \in \Sigma^{2}\right)$.

Since the set of CQLFs for a given switched system can be thought of as a semi-algebraic set, we interpret Parrilo's result in this light.

Theorem IV.1 ([8]). Consider a system of polynomial equalities and inequalities. Then, the search for bounded degree Positivstellensatz refutations can be done using semi-definite programming. If the degree bound is chosen to be large enough, then the SDP will be feasible, and the certificates obtained from its solution.

Parrilo's proof outlines an algorithm that can answer the emptiness question using SOSTOOLS, a MATLAB package which converts an SoS program to an SDP and solves it. The algorithm in his proof will be demonstrated with the following example.

\section{AN EXAMPLE}

Let

$$
A_{1}=\left[\begin{array}{cc}
-0.2 & 0.3 \\
0 & -0.1
\end{array}\right] \text { and } A_{2}=\left[\begin{array}{cc}
-0.9 & 0.4 \\
1.7 & -0.9
\end{array}\right],
$$

which are stable matrices, and consider the arbitrarily switched system $\dot{x}=A_{i} x, i \in\{1,2\}$. We will use the methods outlined in this paper to show the nonexsistence of a CQLF for the switched system.

Analogous to Example II.1, for $V=x^{T} P x$ to be a CQLF, we need $P=P^{T}>0$ and $A_{i}^{T} P+P A_{i} \leq 0$ for $i=1,2$. Hence, the associated system of polynomial inequalities (in the form required for the Positivstellensatz) is

$$
\begin{aligned}
f_{1}(x, y, z):=x & \geq 0, \\
f_{2}(x, y, z):=x z-y^{2} & \geq 0, \\
f_{1}^{A_{1}}(x, y, z):=0.4 x & \geq 0, \\
f_{2}^{A_{1}}(x, y, z):=0.09 x^{2}+0.06 x y & \\
-0.08 x z+0.09 y^{2} & \geq 0, \\
f_{1}^{A_{2}}(x, y, z):=1.8 x-3.4 y & \geq 0, \\
f_{2}^{A_{2}}(x, y, z):=0.16 x^{2}-1.44 x y-1.88 x z & \\
+1.44 x+3.24 y^{2}-2.72 y+2.89 z^{2} & \geq 0 .
\end{aligned}
$$

The non-equalities needed to obtain strict inequalities above are

$$
\begin{array}{r}
g_{1}(x, y, z):=x \neq 0, \\
g_{2}(x, y, z):=x z-y^{2} \neq 0 .
\end{array}
$$

By the Positivstellensatz, we seek a $g \in \operatorname{monoid}\left\{g_{1}, g_{2}\right\}$ and $f \in \operatorname{cone}\left\{f_{1}, f_{2}, f_{1}^{A_{1}}, f_{2}^{A_{1}}, f_{1}^{A_{2}}, f_{2}^{A_{2}}\right\}$ such that $f+g^{2}=0$. Set $g:=g_{1}^{m} g_{2}^{m}$, where the parameter $m$ is chosen to be 1 in this example. If the semi-algebraic set defined by (V.1), (V.2) is indeed empty, Theorem IV.1 ensures that we will find a "certificate of emptiness" provided we search through enough of the cone of SoS polynomials; increasing the parameter $m$ accomplishes this.

Next, we write the general form for an element in cone $\left\{f_{1}, f_{2}, f_{1}^{A_{1}}, f_{2}^{A_{1}}, f_{1}^{A_{2}}, f_{2}^{A_{2}}\right\}$ and set it equal to $f$. Recall from earlier that this can be thought of as a linear combination whose coefficients are SoS polynomials $s_{i}(x, y, z)$ and whose 'unknowns' are all possible multiples of the set $\left\{f_{1}, f_{2}, f_{1}^{A_{1}}, f_{2}^{A_{1}}, f_{1}^{A_{2}}, f_{2}^{A_{2}}\right\}$ without repetition. If the set has $n$ elements, then the number of terms in this $f$ polynomial will be $\left(\begin{array}{l}n \\ 1\end{array}\right)+\left(\begin{array}{l}n \\ 2\end{array}\right)+\cdots+\left(\begin{array}{l}n \\ n\end{array}\right)+1$. For brevity, we refrain from writing out the expansion of $f$, but keep in mind that every summand of the polynomial is multiplied by a $s_{i}(x, y, z)$.

Using sostools, we implement the following SoS program:

$$
\begin{aligned}
& \text { Find SoS polynomials } s_{i}(x, y, z) \text { such that } \\
& \qquad f+\left(g_{1} g_{2}\right)^{2}=0 .
\end{aligned}
$$

In doing so, SoS-variables are called, which are polynomials written in a general basis representation up to a certain degree, with unknown coefficients. This degree must be larger than or equal to the degree of $g$, which is $3 m$; for this example $d=4$. Sostools will then search through the cone of SoS polynomials for $s_{i}(x, y, z)$ satisfying the equality constraint. By the Positivstellensatz, if the degree bound $d$ is sufficiently large, the program will return SoS polynomials certifying the emptiness of the semi-algebraic set defined by (V.1), (V.2). This in turn establishes the nonexistence of a CQLF for this switched system. 
Running Sostools, we find $s_{i}(x, y, z) \in \Sigma^{2}$ such that

$$
\begin{aligned}
f+g^{2} & =-4.4634 \times 10^{-15} x^{6}-3.2535 \times 10^{-15} x^{5} y \\
& +2.9421 \times 10^{-15} x^{5} z-7.1419 \times 10^{-15} x^{4} y^{2} \\
& -1.4048 \times 10^{-13} x^{4} y z+5.0505 \times 10^{-13} x^{4} z^{2} \\
& -4.215 \times 10^{-14} x^{3} y^{3}+7.5615 \times 10^{-13} x^{3} y^{2} z \\
& -8.9199 \times 10^{-14} x^{3} y z^{2}-3.7967 \times 10^{-14} x^{3} z^{3} \\
& +4.035 \times 10^{-13} x^{2} y^{4}-1.0967 \times 10^{-13} x^{2} y^{3} z \\
& -4.817 \times 10^{-14} x^{2} y^{2} z^{2}-3.1445 \times 10^{-14} x^{2} y z^{3} \\
& -8.9278 \times 10^{-15} x^{2} z^{4}-6.9118 \times 10^{-15} x y^{5} \\
& -2.2009 \times 10^{-14} x y^{4} z-2.9263 \times 10^{-14} x y^{3} z^{2} \\
& +2.0733 \times 10^{-14} x y^{2} z^{3}-4.4062 \times 10^{-15} x y z^{4} \\
& -4.8008 \times 10^{-16} x z^{5}+3.4868 \times 10^{-15} y^{6} \\
& -1.8436 \times 10^{-15} y^{5} z-1.224 \times 10^{-14} y^{4} z^{2} \\
& -1.2395 \times 10^{-14} y^{3} z^{3}+1.5697 \times 10^{-15} y^{2} z^{4} \\
& +4.2143 \times 10^{-16} y z^{5}+2.4135 \times 10^{-16} z^{6} \\
& \approx 0 .
\end{aligned}
$$

Given the relatively simple structure of the program, we could scale this type of SoS program to include as many switches (and therefore, matrices) as we like.

\section{SUMMARY AND CONCLUSIONS}

To recapitulate, the idea of this paper was establish the nonexistence of a CQLF for a switched system with the plan shown in Figure 1 below.

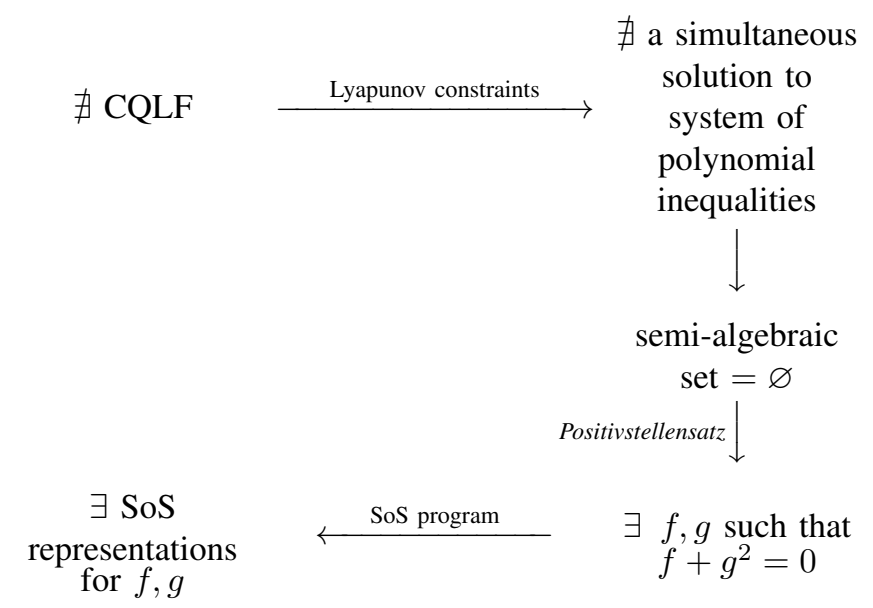

Figure 1. The algorithm presented here.

We translated the problem of determining the existence of a CQLF into a problem involving the simultaneous solution to a system of polynomial inequalities. We then recast that as a problem of determining whether the associated semi-algebraic set was empty. We resolved this "set emptiness question" via the Positivstellensatz and did so by looking for $f$ and $g$ with the desired SoS representations.
We have shown that if there does not exist a CQLF for a given a switched system $\dot{x}=A_{i} x$, we can use SoS programming to provide a certificate of nonexistence. Although the nonexistence of a quadratic Lyapunov function does not necessarily imply that the system is unstable, our result does allow us to definitively rule out the existence of a quadratic Lyapunov function when we suspect one does not exist.

\section{ACKNOWLEDGEMENTS}

This work was supported by National Science Foundation grant CMMI \#0726996.

\section{REFERENCES}

[1] A.A. Agrachev and D. Liberzon, Lie-algebraic stability criteria for switched systems, SIAM Journal on Control and Optimization 40 (2001), 253-269.

[2] R. Horn and C. Johnson, Matrix Analysis, Cambridge University Press, 1985.

[3] C. King and R. Shorten, A singularity test for the existence of common quadratic Lyapunov functions for pairs of stable LTI systems, in Proceeding of the 2004 American Control Conference, 2004, pp. 3881-3884.

[4] C. King and R. Shorten, Singularity conditions for the non-existence of a common quadratic Lyapunov function for pairs of third order linear time invariant dynamic systems, Linear Algebra Appl. vol. 413, no. 1, pp. 24-35, 2006.

[5] D. Liberzon, Switching in Systems and Control, Birkhauser, Basel, 2003.

[6] K.S. Narendra and J. Balakrishnan, A common Lyapunov function for stable LTI systems with commuting A-matrices, IEEE Trans. Automat. Control vol. 39, pp. 2469-2471, 1994.

[7] A. Olshevsky and J.N. Tsitsiklis, On the Nonexistence of Quadratic Lyapunov Functions for Consensus Algorithms, IEEE Transactions on Automatic Control, vol. 53, no. 11, pp. 2642-2645, December 2008.

[8] P.A. Parrilo, Semidefinite programming relaxations for semialgebraic problems, Mathematical Programming Ser. B, vol. 96, no.2, pp. 293-320, 2003.

[9] P.A. Parrilo, "Structured semidefinite programs and semialgebraic geometry methods in robustness and optimization." $\mathrm{PhD}$ thesis, California Institute of Technology, May 2000.

[10] G. Stengle, A nullstellensatz and a positivstellensatz in semialgebraic geometry, Math. Ann. vol. 207, 87-97, 1974. 